\title{
Liver X Receptor $\beta$ Related to Tumor Progression and Ribosome Gene Expression in Papillary Thyroid Cancer
}

\author{
Seonhyang Jeong ${ }^{1}$, In-Kyu Kim², Hyunji Kim², Moon Jung Choi ${ }^{2}$, Jandee Lee², Young Suk Jo ${ }^{1,3}$ \\ ${ }^{1}$ Department of Internal Medicine, Yonsei University College of Medicine, Seoul; ${ }^{2}$ Department of Surgery, Open NBI \\ Convergence Technology Research Laboratory, Severance Hospital, Yonsei Cancer Center, Yonsei University College of \\ Medicine, Seoul; ${ }^{3}$ Brain Korea 21 PLUS Project for Medical Science, Yonsei University, Seoul, Korea
}

Background: Intracellular lipid deposition has been reported in thyroid glands in obese animal and human. To understand the regulatory mechanism of lipid metabolism in thyroid cancer, we investigated the expression status of liver X receptor (LXR) and analyzed its clinicopathological characteristics and molecular biological features.

Methods: Expression status of LXR and its transcriptional targets in human cancers were analyzed using The Cancer Genome Atlas (TCGA). The gene-sets related to high $L X R \beta$ expression was investigated by gene set enrichment analysis (GSEA) using Kyoto Encyclopedia of Genes and Genomes (KEGG) signaling pathways and gene ontology biologic process. Quantitative reverse transcription polymerase chain reaction was performed in thyroid cancer samples using our validation cohort.

Results: In contrast to low expression of $L X R \alpha, L X R \beta$ was highly expressed in thyroid cancer compared to the other types of human cancers. High $L X R \beta$ expression was correlated with the expression of $L X R \beta$ transcriptional targets genes, such as apolipoprotein $\mathrm{C} 1$ (APOC1), APOC2, apolipoprotein E (APOE), ATP binding cassette subfamily G member 8 (ABCG8), sterol regulatory elementbinding protein 1c (SREBP1c), and SPOT14. Furthermore, High $L X R \beta$ expression group indicated poor clinicopathological characteristics and aggressive molecular biological features independently from the drive mutation status. Mechanistically, high $L X R \beta$ expression was coordinately related to ribosome-related gene sets.

Conclusion: The mechanistic link between $L X R \beta$ and ribosomal activity will be addressed to develop new diagnostic and therapeutic targets in thyroid cancers.

Keywords: Thyroid neoplasms; Obesity; Metabolism; Prognosis; Liver X receptors; Ribosomes

\section{INTRODUCTION}

Thyroid cancer is the most common endocrine malignancy
$[1,2]$. The incidence of thyroid cancer has strikingly increased in the last couple of decades, especially in Korea [3]. The cause of this increase might be the early detection of differentiated
Received: 2 April 2020, Revised: 15 June 2020, Accepted: 26 June 2020

Corresponding authors: Jandee Lee

Department of Surgery, Open NBI Convergence Technology Research Laboratory, Severance Hospital, Yonsei Cancer Center, Yonsei University College of Medicine, 50-1 Yonsei-ro, Seodaemun-gu, Seoul 03722, Korea Tel: 82-2-2228-0830, Fax: +82-2-2227-8129, E-mail: JANDEE@yuhs.ac

Young Suk Jo

Department of Internal Medicine, Yonsei University College of Medicine,

50-1 Yonsei-ro, Seodaemun-gu, Seoul 03722, Korea

Tel: +82-2-2228-0752, Fax: +82-2-393-6884, E-mail: joys@yuhs.ac
Copyright (C) 2020 Korean Endocrine Society

This is an Open Access article distributed under the terms of the Creative Commons Attribution Non-Commercial License (https://creativecommons.org/ licenses/by-nc/4.0/) which permits unrestricted non-commercial use, distribution, and reproduction in any medium, provided the original work is properly cited. 
thyroid cancer, mainly papillary thyroid microcarcinoma, by the introduction of high-sensitivity neck ultrasound [4,5]. Although an increase in the incidence of thyroid cancer has been postulated, its underlying cause has not been well-recognized $[6,7]$.

The most well-known etiology of thyroid cancer is radiation exposure $[8,9]$. However, radiation-related papillary thyroid carcinoma (PTC) especially occurred after the Chernobyl nuclear accident caused RET/PTC rearrangements, whereas in Korea most of the PTC cases report B-Raf proto-oncogene, serine/ threonine kinase $(B R A F) \mathrm{V} 600 \mathrm{E}$ mutation as its driver mutation [10-12]. In addition, there is no strong evidence to support that Korean individuals are subjected to increased radiation exposure [13]. Recently, other causative factors, such as iodine intake and obesity, have also been suggested [14-16]. However, there is also no evidence of increased iodine intake in the Korean population. Obesity is likely to be associated with an increased incidence of thyroid cancer in Korea [17,18]. As seen in other developed countries, the prevalence of obesity and metabolic diseases in Korea has been increasing, thus leading to an increase in the incidence of obesity-related cancer [19]. In this context, some epidemiological studies have reported that obesity might be associated with thyroid cancer, but substantial evidence is lacking.

The effects of obesity on the normal thyroid gland in animal models and humans have been reported [14]. Abnormalities in thyroid function and morphology have been observed in genetically modified obese animals such as $o b / o b$ and $d b / d b$ mice; moreover, recent studies have reported that obesity causes lipid accumulation in the thyroid gland of humans and animals $[20,21]$. In addition, a high fat diet generated a more aggressive phenotype of thyroid cancer in Thrb(PV/PV)Pten(+/-) mice [22, 23]. In human thyroid cancer, the body mass index has been suggested as a factor related to aggressive tumor behavior $[24,25]$. Moreover, an oncocytic variant of PTC was reported to be associated with obesity [26]. These data from studies in mice and humans collectively suggest that obesity might affect the normal thyroid physiology and thyroid cancer behavior. However, although an association between obesity and thyroid cancer has been suggested, the role of lipid metabolism in thyroid cancer remains to be elucidated $[18,23]$.

Liver X receptor (LXR), recently deorphanized by the discovery of oxysterol as its endogenous ligand, is a member of the nuclear receptor family [27-29]. Liver X receptors ( $L X R \alpha$ and $L X R \beta)$ are important regulators of cholesterol and lipid metabolism [30,31]. In this study, we investigated the expression pattern of $L X R \alpha$ and $L X R \beta$ in human thyroid cancer to improve our current understanding of lipid metabolism in thyroid cancer.

\section{METHODS}

\section{Public data acquisition}

Subsets of normalized raw data for RNA expression (RNA reads per kilobase per million [RPKM] converted to transcripts per million [TPM]) and clinicopathological features were provided by The Cancer Genome Atlas (TCGA, https://tcga-data.nci.nih. gov/tcga/). Using these data sets, twelve types of cancer were analyzed for their mRNA expression status of $L X R \alpha$ and $L X R \beta$ : breast invasive carcinoma (BRCA; 1,092 cases), cervical squamous cell carcinoma and endocervical adenocarcinoma (CESC; 304 cases), colon adenocarcinoma (COAD; 385 cases), liver hepatocellular carcinoma (LIHC; 361 cases), lung adenocarcinoma (LUAD; 516 cases), ovary serous cystadenocarcinoma (OV; 303 cases), pancreatic adenocarcinoma (PAAD; 147 cases), prostate adenocarcinoma (PRAD; 947 cases), skin cutaneous melanoma (SKCM; 94 cases), stomach adenocarcinoma (STAD; 415 cases), thyroid carcinoma (THCA; 505 cases), and uterine corpus endometrial carcinoma (UCEC; 545 cases). $L X R \alpha$ and $L X R \beta$ expression levels were compared in various types of human cancers and further analyzed in thyroid cancer by comparisons with paired or unpaired normal thyroid samples. In thyroid cancer, the major transcriptional targets of LXR were also analyzed to investigate the relation of $L X R \beta$ expression with the expression of its target genes. The protein expression level was analyzed by calculating the frequency of LXR $\alpha$ - and LXR $\beta$-positive samples provided by Human Protein Atlas (HPA, www.proteinatlas.org) including 12 cancer types: BRCA 11 cases, CESC 12 cases, COAD 11 cases, LIHC 11 cases, LUAD eight cases, OV 11 cases, PAAD 12 cases, PRAD 11 cases, SKCM 12 cases, STAD nine cases, THCA four cases, and UCEC 12 cases. We also selected GSE83520, GSE77039, GSE41223, GSE96545 from the Gene Expression Omnibus (GEO) database as in vitro validation set in addition to the TCGA data.

\section{Patients and tissue samples}

PTC tissue samples along with matched normal tissues from the same patients were collected from patients who underwent thyroidectomy at Yonsei Cancer Center from April to October 2010 (Seoul, South Korea). All tissues were frozen in liquid nitrogen immediately after surgery and stored at $-80^{\circ} \mathrm{C}$ before use. This research has been approved by the Institutional Review Board of Severance Medical Center (Seoul, South Korea) on 30 September 2013 (No. 4-2013-0546); the requirement for informed 
consent was waived because of its retrospective nature of the study.

\section{RNA isolation and quantitative reverse-transcription polymerase chain reaction analysis}

Total RNA was extracted according to the manufacturer's protocol (Trizol reagent, Qiagen, CA, USA). Complementary DNA (cDNA) was synthesized from $1 \mu \mathrm{g}$ of total RNA using the QuantiTect Reverse Transcription Kit (Qiagen). The primers used in this study are as follows: $L X R \beta, 5^{\prime}$-TGC AAC AAA CGC TCC TTC TC-3' and 5'-CCG AGA AGA TGT TGA TGG CG-3'; RPL15, 5'-TAC GGC AAG CCT GTC CAT CAT G-3' and 5'GTA TGT GGA ATC TTC ACC AAC CC-3'; RPL11, 5'-AGA GTG GAG ACA GACTGA CGC G-3' and 5'-CGG ATG CCA AAG GAT CTG ACA G-3'; RPL19, 5'-TCA CAG CCT GTA CCT GAA GGT G-3' and 5'-CGT GCT TCC TTG GTC TTA GAC C-3'; RPS30, 5'-CGT GCT GGA AAA GTG AGA GGT C-3' and 5'-TGC CAA AGG TGG GCA CAA CGT T-3'; glyceraldehyde-3-phosphate dehydrogenase $(G A P D H), 5^{\prime}$-ACC ACA GTC CAT GCC ATC AC-3' and 5'-TCC ACC ACC CTG TTG CTG TA-3'. Reverse transcription-polymerase chain reaction (RT-PCR) was performed on cDNA using the Solg 2X PCR Smart mix Kit (Solgent, Daejeon, Korea). PCR products were separated on $1.0 \%$ agarose gel in $1 \mathrm{X}$ TAE buffer and RedSafe (Intron, Seongnam, Korea) was used for visualization of the PCR band. The image of the gel was confirmed by MINIBIS PRO (DNR, Jerusalem, Israel). All experiments were repeated three times, and each experiment was performed in triplicate.

\section{Gene set enrichment analysis}

Gene set enrichment analysis (GSEA) is software that analyzes microarray and other data with different expressions based on biological information. Enrichment score (ES) values can be calculated using weighted Kolmogorov-Smirnov statistics. The most important gene group can be identified by identifying the location (leading edge subset) where the calculated ES value represents the maximum value. Normalized enrichment score (NES) is obtained by normalizing ES values to gene set size. The expression profile was selected as the top and bottom groups by the top $25 \%$ and bottom $25 \%$, depending on the $L X R \beta$ expression ( $n=126$ for each group). We selected significant gene sets based on the $P$ value $\leq 0.05$ and the false discovery rate (FDR) q-value $<0.25$.

\section{Statistical analyses}

The results of the clinicopathological analyses were expressed as mean \pm standard deviation (SD). Mean comparisons were performed using Student's $t$ test and group comparisons were performed using a 2-tailed chi-square test, Fisher's exact test, or linear-by-linear associations. Pearson's correlation coefficient was used to examine the association of genes. The experimental data are expressed as mean \pm standard error of the mean and comparisons were performed using Mann-Whitney $U$ test. Statistical analysis was performed using Prism (GraphPad Software, SanDiego, CA, USA) and SPSS version 25.0 for Windows (IBM Corp., Armonk, NY, USA). All $P$ values are twosided. Image analyses was conducted by ImageJ (National Institutes of Health) [32].

\section{RESULTS}

\section{Comparisons of $L X R \alpha$ and $L X R \beta$ expression in human cancers}

To investigate the $L X R \alpha$ and $L X R \beta$ expression status in human cancers, we collected and compared the mRNA levels of $L X R \alpha$ and $L X R \beta$ in twelve types of cancers from TCGA. $L X R \alpha$ showed a relatively high expression in liver cancer $(59.88 \pm$ 23.64), but low expression in thyroid cancer (16.36 \pm 8.09 ), suggesting $L X R \alpha$ to be a major isoform of LXRs in liver cancer (Fig. 1A). In addition, pancreatic, lung, colorectal, stomach and breast cancers also showed relatively high expression of $L X R \alpha$. Interestingly, $L X R \beta$ was highly expressed in thyroid cancer (121.6 \pm 27.02$)$ whereas $L X R \beta$ expression was relatively low in liver and breast cancer $(70.73 \pm 28.28$ and $66.29 \pm 24.87$, respectively), also suggesting that $L X R \beta$ might be a dominant isoform of LXRs in thyroid cancer (Fig. 1B). In addition to thyroid cancer, melanoma, endometrial, prostate, cervical and lung cancer samples also showed high $L X R \beta$ mRNA expression. Compatible with our findings from mRNA expression analyses in TCGA, the protein levels analyzed using HPA (Fig. 1C, D) revealed that LXR $\beta$ expression was more frequently observed in thyroid cancer than in other types of human cancers. The mRNA and protein expression levels appeared to be somewhat different in certain types of cancers (Fig. $1 \mathrm{C}, \mathrm{D})$. For example, $L X R \alpha$ mRNA expression in cervical cancer seemed to be relatively low but protein expression of $\mathrm{LXR} \alpha$ was frequently observed (Fig. 1A, C). However, mRNA and protein levels of $L X R \beta$ in thyroid cancer were higher than those in other types of cancers (Fig. 1B, D).

To verify that $L X R \beta$ is a dominant isoform of LXRs in thyroid cancer, we compared the mRNA expression levels of $L X R \alpha$ and $L X R \beta$ between normal thyroid tissues and PTC samples. As 

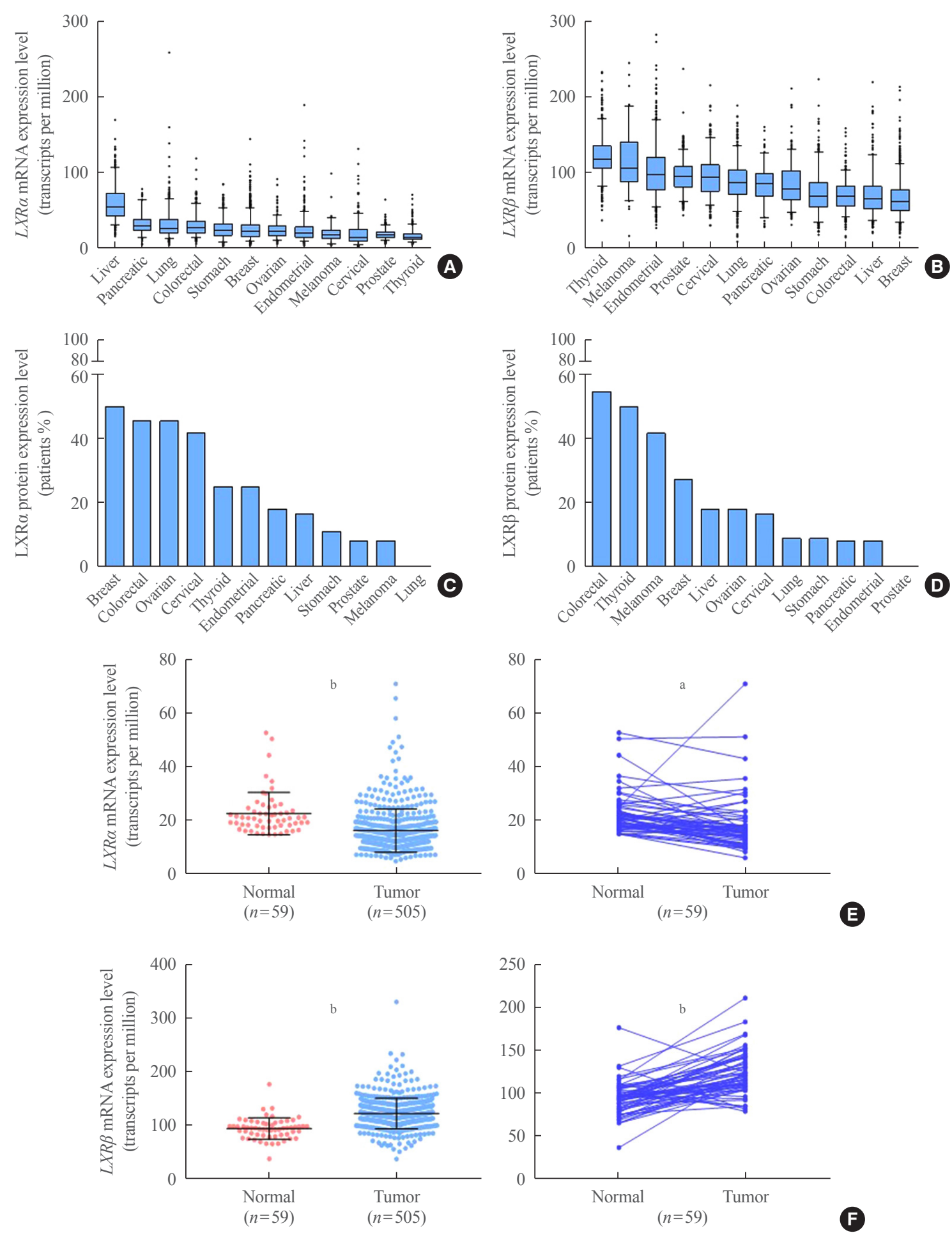

Fig. 1. Comparison of liver X receptor (LXR) expression in human cancers. (A) Comparison of $L X R \alpha$ mRNA expression in various human cancers. (B) Comparison of $L X R \beta$ mRNA expression in various human cancers. (C) Comparison of LXR $\alpha$ protein expression in various human cancers. (D) Comparison of LXR $\beta$ protein expression in various human cancers. (E) Comparison of $L X R \alpha$ mRNA expression between normal tissues and thyroid cancer samples by unpaired (left panel) and paired (right panel) $t$ tests. (F) Comparison of $L X R \beta$ mRNA expression between normal tissues and thyroid cancer samples by unpaired (left panel) and paired (right panel) $t$ tests. Comparison of $L X R \alpha$ and $L X R \beta$ mRNA expression levels was performed using TCGA data $(n=505)$ and comparison of LXR $\alpha$ and LXR $\beta$ protein levels was conducted using Human Protein Atlas Data. Data are presented as mean \pm standard deviation. Mean comparisons were analyzed by an unpaired or paired $t$ test. ${ }^{\mathrm{a}} P<0.01 ;{ }^{\mathrm{b}} P<0.0001$. 
shown in Fig. 1E, $L X R \alpha$ expression in PTC was lower than that in unpaired and paired normal thyroid tissues (unpaired $t$ test, $P<0.0001$; paired $t$ test, $P<0.01$ ). On the contrary, $L X R \beta$ expression in PTC was higher than that in unpaired and paired normal thyroid tissues (unpaired $t$ test, $P<0.0001$; paired $t$ test, $P<0.0001$ ) (Fig. 1F). Taken together, $L X R \beta$ might be a major isoform of LXRs in thyroid cancer and its expression might be increased in a tumor-specific manner.

\section{Clinicopathological and molecular feature analyses of patients with PTC according to $L X R \boldsymbol{B}$ expression}

To understand the role of increased $L X R \beta$ expression in patients with PTC, we compared the clinicopathological characteristics of the patients according to $L X R \beta$ expression levels (Table 1). For this analysis, we divided THCA data into four groups according to $L X R \beta$ expression and used the upper and lower quartiles as groups with high and low $L X R \beta$ expression, respectively. Interestingly, male patients were more frequently observed in the group with high $L X R \beta$ expression. In addition, TNM stage was more advanced in the group with high $L X R \beta$ expression. These data suggested that $L X R \beta$ expression might be related to aggressive clinical features of PTC. In line with clinicopathological analyses, we also compared the molecular features of patients with PTC according to the $L X R \beta$ expression levels (Table 2). A high mRNA cluster number, which has been known to be associated with aggressive molecular features, was related to high $L X R \beta$ expression. Interestingly, although the frequency of RAS proto-oncogene, GTPase $(R A S)$ and $B R A F$ driver mutations was not different between the two groups, the BRAF/RAS score was lower in the group with high $L X R \beta$ expression, suggesting that this group has BRAF-like features, a molecular feature of aggressive thyroid cancer. In support of this finding, the differentiation score was also lower in the group with high $L X R \beta$ expression $(P<0.0001)$. In summary, these clinicopathological and molecular profile analyses suggest that $L X R \beta$ might have a role in tumor progression of patients with PTC.

\section{Transcriptional target analyses of $L X R \beta$ in PTC}

To gain a deeper understanding of the molecular mechanisms by which $L X R \beta$ influences tumor behavior, we performed correlation analyses between the expression status of $L X R \beta$ and its 24 well-known target genes regulating lipid metabolism. Consistent with previous reports, this analysis indicated that $L X R \beta$ expression was correlated with the expression of $L X R \beta$ target genes [33], especially apolipoprotein $\mathrm{C} 1$ (APOC1), APOC2, apolipoprotein $\mathrm{E}(A P O E)$, ATP binding cassette subfamily $\mathrm{G}$ member $8(A B C G 8)$, sterol regulatory element-binding protein 1c (SREBP1c; sterol regulatory element binding transcription

Table 1. Clinicopathological Characteristics of TCGA THCA According to the $L X R \beta$ Expression Status

\begin{tabular}{|c|c|c|c|}
\hline \multirow[b]{2}{*}{ Characteristic } & \multicolumn{2}{|c|}{$L X R \beta$ expression status } & \multirow[b]{2}{*}{$P$ value } \\
\hline & $\begin{array}{l}\text { Low expression } \\
\quad(n=126)\end{array}$ & $\begin{array}{l}\text { High expression } \\
\quad(n=126)\end{array}$ & \\
\hline Age, yr & $47.89 \pm 15.00$ & $49.55 \pm 16.21$ & $0.400^{\mathrm{a}}$ \\
\hline Sex, male:female & $30(23.8): 96(76.2)$ & $45(35.7): 81(64.3)$ & $0.039^{\mathrm{b}}$ \\
\hline Tumor size, cm & $3.04 \pm 1.69$ & $3.22 \pm 1.62$ & $0.446^{\mathrm{a}}$ \\
\hline MACIS score & $5.28 \pm 1.41$ & $5.65 \pm 1.61$ & $0.076^{\mathrm{a}}$ \\
\hline Histologic subtype & & & $0.133^{\mathrm{b}}$ \\
\hline Follicular variant & $30(24.2)$ & $28(22.4)$ & \\
\hline Conventional & $88(71.0)$ & $81(64.8)$ & \\
\hline Tall cell variant & $5(4.0)$ & $13(10.4)$ & \\
\hline Others $^{\mathrm{c}}$ & $1(0.8)$ & $3(2.4)$ & \\
\hline \multicolumn{2}{|c|}{ Extrathyroidal extension } & & $0.422^{\mathrm{b}}$ \\
\hline No & $80(70.2)$ & $73(65.2)$ & \\
\hline Yes & $34(29.8)$ & $39(34.8)$ & \\
\hline Multifocality & & & $0.520^{\mathrm{b}}$ \\
\hline No & $62(50.0)$ & $66(54.1)$ & \\
\hline Yes & $62(50.0)$ & $56(45.9)$ & \\
\hline T stage & & & $0.232^{\mathrm{b}}$ \\
\hline $\mathrm{T} 1$ & $37(31.6)$ & $29(25.2)$ & \\
\hline $\mathrm{T} 2$ & $36(30.8)$ & $37(32.2)$ & \\
\hline $\mathrm{T} 3$ & $40(34.2)$ & $42(36.5)$ & \\
\hline $\mathrm{T} 4$ & $4(3.4)$ & $7(6.1)$ & \\
\hline N stage & & & $0.370^{\mathrm{b}}$ \\
\hline No & $56(53.8)$ & $52(47.7)$ & \\
\hline N1 & $48(46.2)$ & $57(52.3)$ & \\
\hline M stage & & & $0.159^{b}$ \\
\hline M0 & $60(98.4)$ & $55(93.2)$ & \\
\hline M1 & $1(1.6)$ & $4(6.8)$ & \\
\hline TNM stage & & & $0.049^{\mathrm{b}}$ \\
\hline I & $68(58.1)$ & $59(50.9)$ & \\
\hline II & $15(12.8)$ & $9(7.8)$ & \\
\hline III & $27(23.1)$ & $31(26.7)$ & \\
\hline IV & $7(6.0)$ & $17(14.7)$ & \\
\hline
\end{tabular}

Values are expressed as mean \pm standard deviation or number (\%). TCGA, The Cancer Genome Atlas; THCA, thyroid carcinoma; $L X R \beta$, liver $\mathrm{X}$ receptor $\beta$; MACIS, metastases, age, completeness of resection, invasion, and size; TNM, tumor, node, metastasis.

${ }^{\mathrm{a}} P$ values calculated by Student's $t$ test; ${ }^{\mathrm{b}} P$ values calculated by chisquare test or linear-by-linear association; ${ }^{\mathrm{c} O t h e r s ~ i n c l u d e d ~ t h e ~ r a r e ~}$ variants of papillary thyroid carcinoma such as columnar cell variant, diffuse sclerosing variant, cribriform-morular variant, and so forth. 
factor 1 [SREBF1]), and thyroid hormone responsive (THRSP, SPOT14) (Fig. 2A). Among these positively correlated genes, $A P O E$ and $S R E B P 1 C$ showed positive correlation co-efficiency (Pearson $R=0.31, P<0.0001$; and Pearson $R=0.30, P<0.0001$,

Table 2. Molecular Features of TCGA THCA According to the $L X R \beta$ Expression Status

\begin{tabular}{|c|c|c|c|}
\hline \multirow[b]{2}{*}{ Characteristic } & \multicolumn{2}{|c|}{$L X R \beta$ expression } & \multirow[b]{2}{*}{$P$ value } \\
\hline & $\begin{array}{c}\text { Low } \\
\text { expression } \\
(n=126)\end{array}$ & $\begin{array}{c}\text { High } \\
\text { expression } \\
(n=126)\end{array}$ & \\
\hline
\end{tabular}

mRNA cluster number $<0.0001^{\text {b }}$

$\begin{array}{ccc}1 & 49(39.8) & 29(24.2) \\ 2 & 23(18.7) & 6(5.0) \\ 3 & 17(13.8) & 14(11.7) \\ 4 & 23(18.7) & 31(25.8) \\ 5 & 11(8.9) & 40(33.3)\end{array}$

miRNA cluster number

1

2

$5(4.0)$

$5(4.0)$

$30(24.2)$

$35(28.2)$

3

$15(12.1)$

$25(20.2)$

4

$37(29.8)$

$27(21.8)$

$17(13.7)$

$19(15.3)$

6

$20(16.1)$

$13(10.5)$

Ras mutation

$\begin{array}{lrc}\text { Absent } & 108(87.1) & 115(92.7) \\ \text { Present } & 16(12.9) & 9(7.3)\end{array}$

BRAF mutation

$\begin{array}{lll}\text { Absent } & 70(55.6) & 65(52.0) \\ \text { Present } & 56(44.4) & 60(48.0)\end{array}$

TERT promoter mutation

$\begin{array}{lccr}\text { Absent } & 96(91.4) & 75(85.2) & \\ \text { Present } & 9(8.6) & 13(14.8) & \\ \text { BRAF/RAS score } & -0.10 \pm 0.77 & -0.36 \pm 0.65 & 0.014^{\mathrm{a}} \\ \text { ERK score } & 0.95 \pm 22.34 & 6.64 \pm 18.81 & 0.060^{\mathrm{a}} \\ \text { Differentiation score } & 0.35 \pm 1.18 & -0.25 \pm 1.04 & <0.0001^{\mathrm{a}} \\ \text { Tumor status } & & & 0.131^{\mathrm{b}} \\ \text { Free } & 98(88.3) & 109(94.0) & \\ \text { With tumor } & 13(11.7) & 7(6.0) & \\ & & & \end{array}$

Values are expressed as number (\%) or mean \pm standard deviation. TCGA, The Cancer Genome Atlas; THCA, thyroid carcinoma; $L X R \beta$, liver $\mathrm{X}$ receptor $\beta$; BRAF, B-Raf proto-oncogene; TERT, telomerase reverse transcriptase; RAS, RAS proto-oncogene GTPase; ERK, mitogen-activated protein kinase 1 .

${ }^{\mathrm{a}} P$ values calculated by Student's $t$ test; ${ }^{\mathrm{b}} P$ values calculated by chisquare test or linear-by-linear association. respectively). To support the results of our correlation analyses, we compared the expression of the target genes between normal thyroid tissues and cancer samples. Four out of six representative target genes, including APOC1, APOE, SREBP1c, and SPOT14 showed increased expression in cancer samples compared with that in their unpaired (Fig. 2B) and paired normal tissues (data not shown). In line with our correlation analyses, all six representative target genes showed high expression patterns in the group with high $L X R \beta$ expression; $A P O C 1, A P O E$, and $S R E B P 1 C$ showed a strong statistical significance (Fig. 2C).

After confirming the positive relationship of $L X R \beta$ with its target genes, we investigated the other genes correlated with $L X R \beta$ by GSEA using TCGA THCA. Using gene sets provided by Kyoto Encyclopedia of Genes and Genomes (KEGG) signaling pathways, two gene sets (base excision repair and ribosome) were coordinately enriched in the group with high $L X R \beta$ expression. Interestingly, using gene sets listed by the gene ontology (GO) term biological process, all gene sets enriched in the group with high $L X R \beta$ expression were related to the ribosome, such as large ribosomal subunit, ribosomal subunit, ribosome and ribosome assembly (Fig. 3A). Taken together, GSEA using two representative lists of gene sets consistently indicated that high $L X R \beta$ expression was correlated with high expression of ribosome-related genes. Examination of individual genes related to ribosomes in KEGG and GO gene lists indicated that ribosomal protein L15 (RPL15), RPL11, RPL19, and ribosomal protein S30 (RPS30) were the significantly up-regulated genes (Fig. 3B).

To verify our findings, we determined the Pearson correlation coefficient between these genes, which showed a positive relationship of $L X R \beta$ with RPL15, RPL11, RPL19, and RPS30 (Fig. $4 \mathrm{~A})$. In addition, the selected target gene was found to be increased in tumor than normal through THCA, GSE83520 data set (Fig. 4B). To verify a positive relationship of $L X R \beta$ with RPL15, RPL11, RPL19, and RPS30 in vitro, we collected in vitro datasets from GEO and analyzed GSE77039 (LXR agonist), GSE41223 (LXR agonist), and GSE96545 (LXR knockout mice). All these in vitro data analyses consistently supported our findings (Supplemental Fig. S1). Using our own validation sets from PTC patients who underwent thyroidectomy, $L X R \beta$ expression was up-regulated in cancer samples compared to that in matched contra-lateral normal tissues. In addition, RPL15, RPL11, RPL19, and RPS30 expression was also increased in cancer samples; this was confirmed by our quantitative analyses using Image J (Fig. 4C, D). 

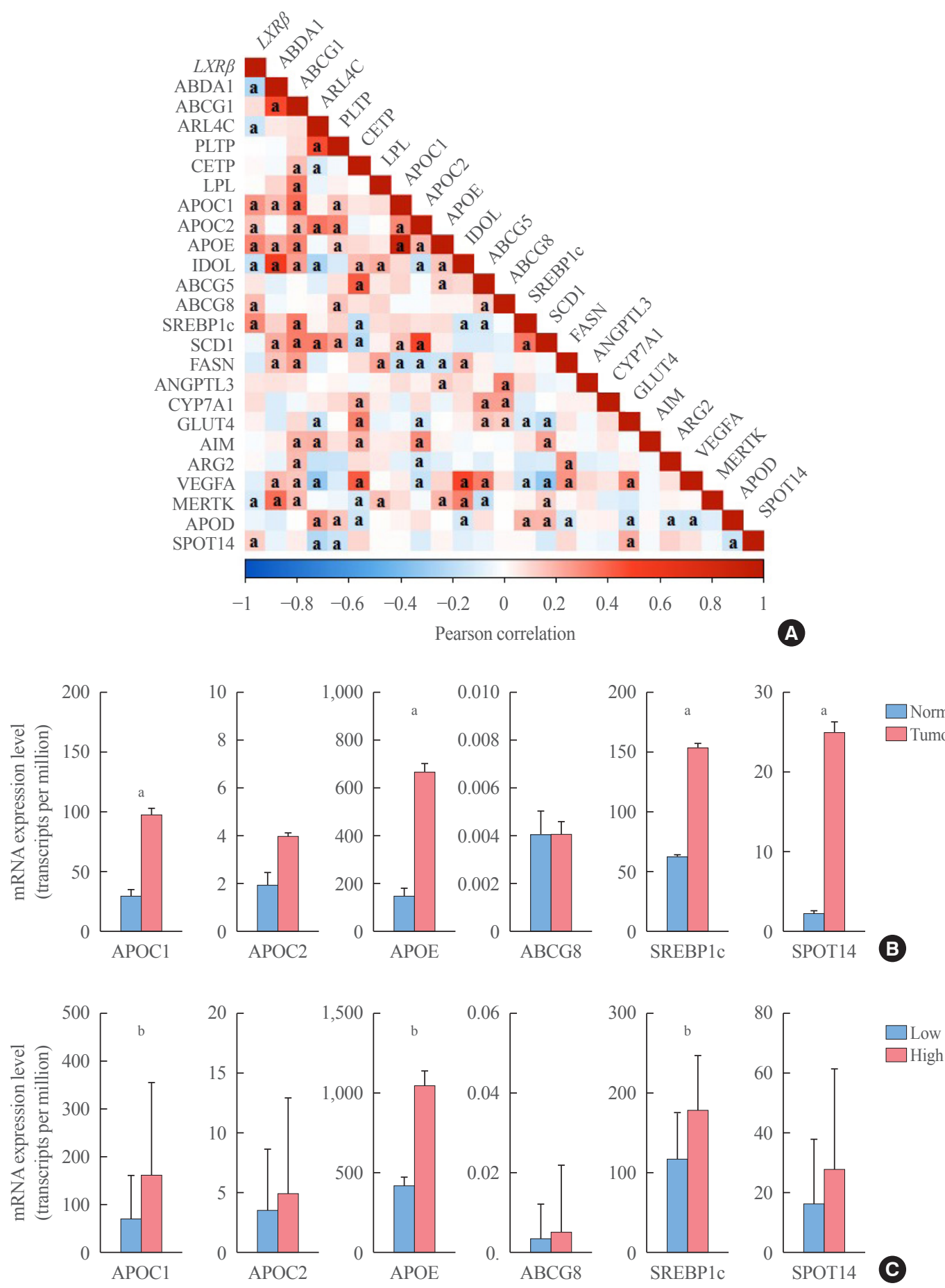

Fig. 2. Correlation analysis between expression of liver $X$ receptor $\beta(L X R \beta)$ and its transcriptional target genes. (A) Correlation between expression of $L X R \beta$ and its transcriptional target genes in The Cancer Genome Atlas (TCGA) thyroid carcinoma (THCA). Correlation coefficients were calculated by Pearson's method. (B) Comparison of mRNA expression level of $L X R \beta$ representative target genes between normal and cancer tissues in TCGA THCA. (C) Comparison of mRNA expression level of $L X R \beta$ representative target genes according to $L X R \beta$ expression status ( $n=126$ for each group). Data are presented as mean \pm standard deviation. Mean comparisons were analyzed by an unpaired $t$ test. APOC1, apolipoprotein $\mathrm{C} 1$; APOC2, apolipoprotein $\mathrm{C} 2$; APOE, apolipoprotein E; ABCG8, ATP binding cassette subfamily G member 8; SREBP1c, sterol regulatory element-binding protein 1c; SPOT14, thyroid hormone responsive (THRSP). ${ }^{a} P<0.01 ;{ }^{b} P<0.0001$. 

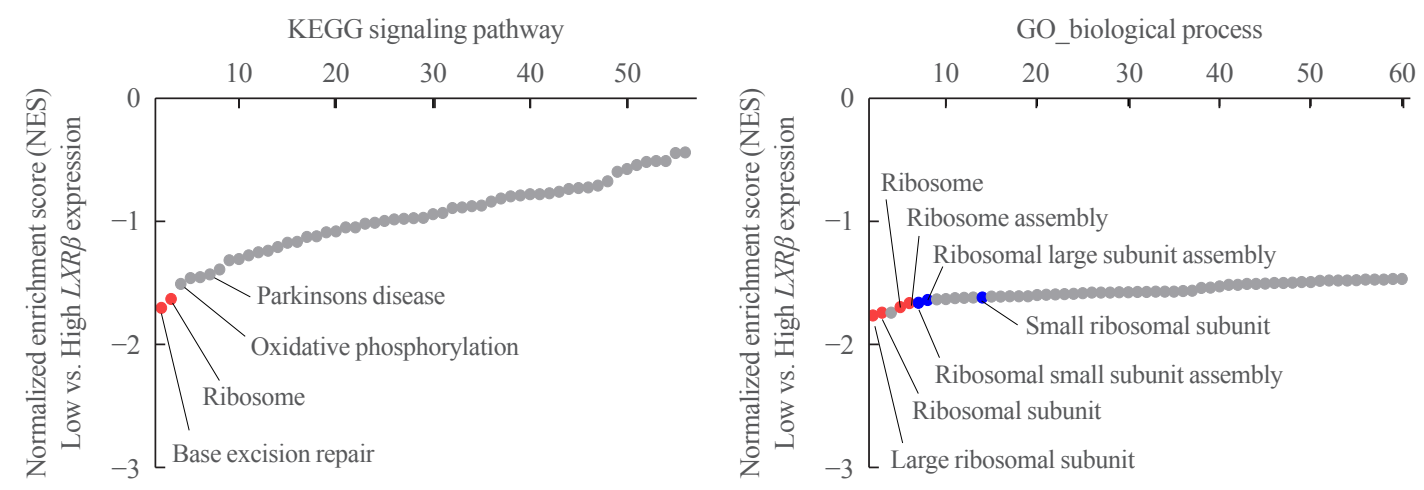

(A)

\section{KEGG RIBOSOME}

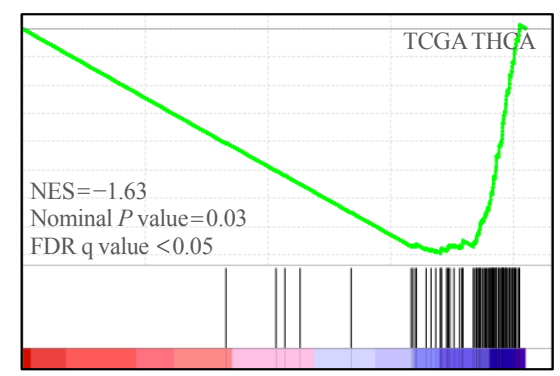

Low $L X R \beta$

$\operatorname{High} L X R \beta$
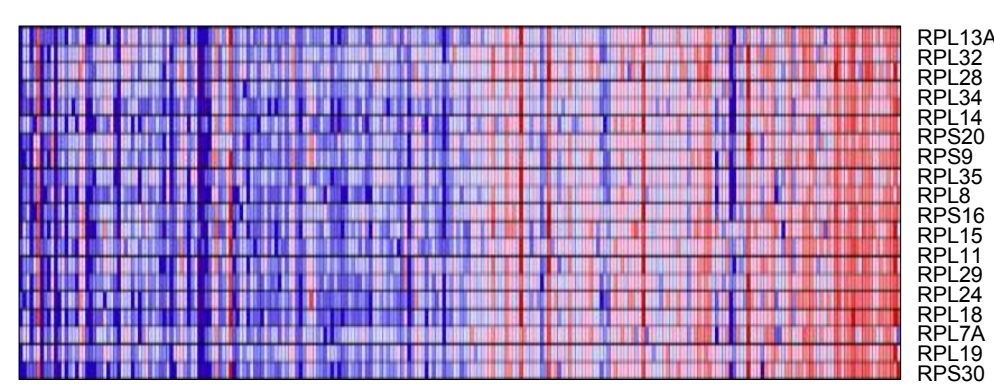

Low $L X R \beta$

$\operatorname{High} L X R \beta$

GO_RIBOSOME

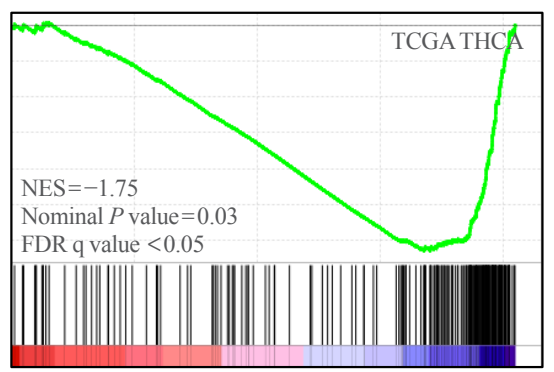

Low $L X R \beta$

High $L X R \beta$
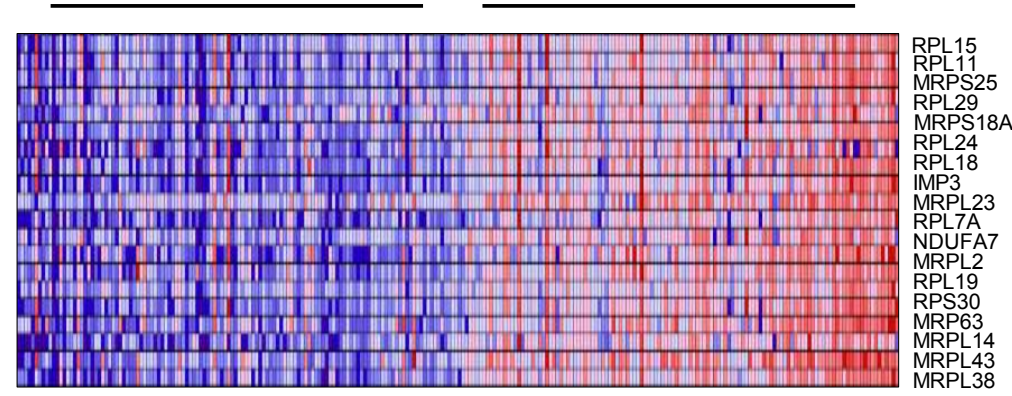

Low $L X R \beta$

High $L X R \beta$

Fig. 3. Correlation of liver $\mathrm{X}$ receptor $\beta(L X R \beta)$ with the expression of ribosome gene sets. (A) Representative results of gene set enrichment analysis (GSEA) using Kyoto Encyclopedia of Genes and Genomes (KEGG) signaling pathways and gene ontology (GO) term (biological process) in thyroid carcinoma (THCA) according to $L X R \beta$ expression status (low vs. high $L X R \beta$ expression group ( $n=126$ for each group). Gene sets of interest are indicated by the name of gene sets. Red circles indicate statistically significant gene sets (nominal $P$ value $\leq 0.05$ and false discovery rate q-value $\leq 0.25$ ). Blue circles indicate gene sets with high FDR q-values although the $P$ value seems meaningful (nominal $P$ value $\leq 0.05$ and FDR q-value $>0.25$ ). (B) Detailed information of KEGG ribosome and GO ribosome gene sets coordinately enriched in the group with high $L X R \beta$ expression, with the list of gene names. NES, normalized enrichment score.

\section{DISCUSSION}

The incidence of obesity and obesity-related diseases, such as dyslipidemia and diabetes mellitus, has been increasing worldwide [34]. Obesity has been suggested as a cause of various cancers such as colorectal cancer, endometrial cancer, and esopha- geal cancer [15]. Various mechanisms have been suggested to explain how these obesity-related cancers develop. The most studied biological mechanisms are those related to insulin and insulin-like growth factors, sex steroid and sex-steroid binding globulin, and adipokines such as adiponectin and leptin [35]. In addition, obesity-related inflammatory cytokines, nuclear factor 

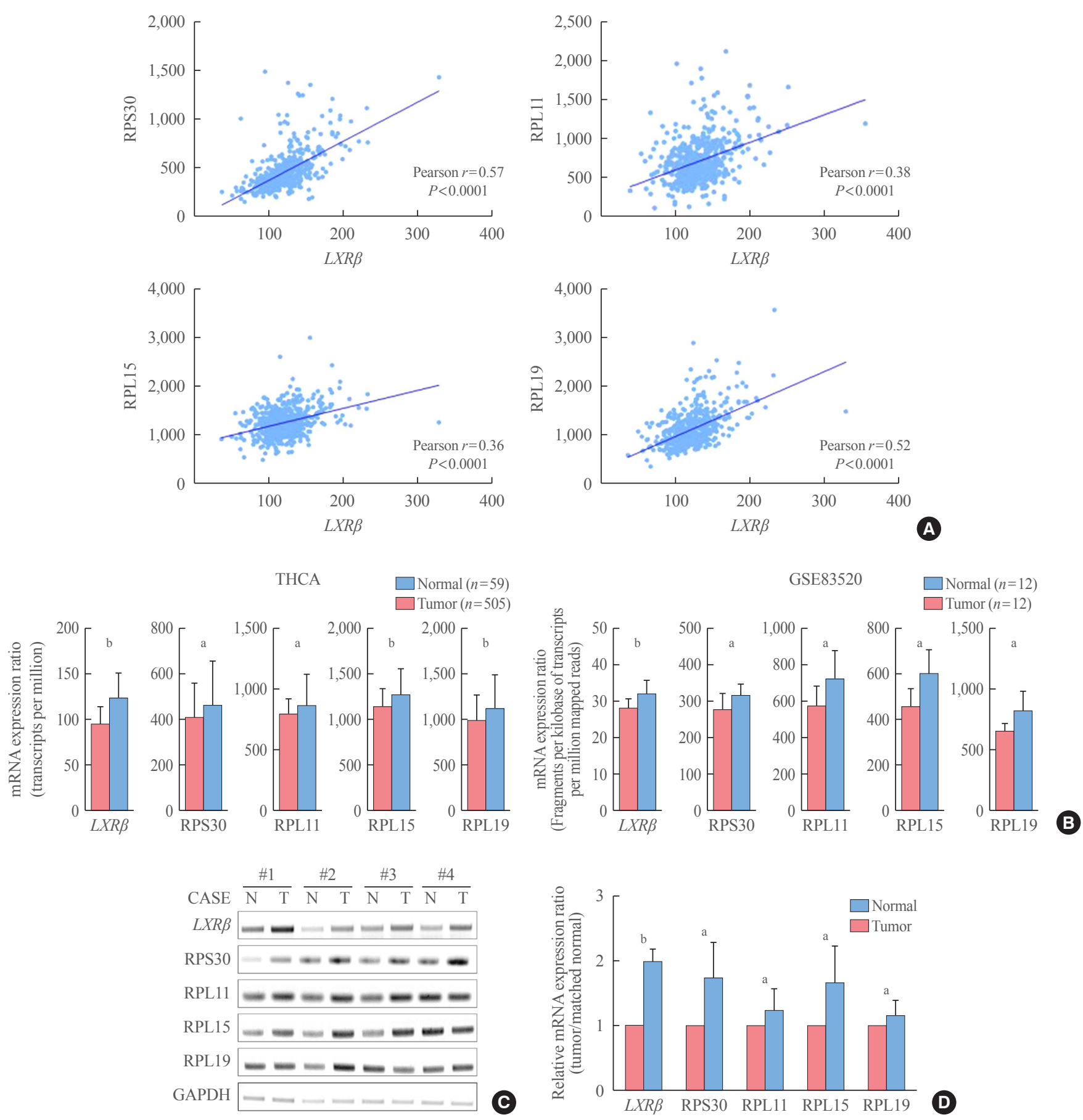

Fig. 4. Positive relationship of liver X receptor $\beta(L X R \beta)$ with the expression of representative ribosome genes. (A) Correlation analysis of $L X R \beta$ with representative ribosome genes ( $n=505$, using thyroid carcinoma [THCA] data). Correlation coefficients were calculated by Pearson's method. $P$ values are indicated on the corresponding figures. (B) Selected target gene expressions were identified in THCA and GSE83520 data sets. GSE83520 is the data that performed RNA sequencing with the tumor and normal tissues of 12 papillary thyroid carcinoma patients. (C) Representative figure of reverse transcription-polymerase chain reaction (RT-PCR) validating THCA analysis using our validation samples. All RT-PCR assays were representative of at least three independent experiments. (D) Semi-quantitative analysis of RT-PCR results (each group $n=8$ ) using ImageJ software. Data are presented as mean \pm standard deviation. Mean comparisons were analyzed by Wilcoxon signed rank test. All experiments were repeated three times, and each experiment was performed in triplicate. N, normal; T, tumor; RPS30, ribosomal protein S30; RPL11, ribosomal protein L11; RPL15, ribosomal protein L15; RPL19, ribosomal protein L19; GAPDH, glyceraldehyde-3-phosphate dehydrogenase. ${ }^{\text {a }} P<0.05$; ${ }^{\mathrm{b}} P<0.001$. 
$\kappa \mathrm{B}$ system, altered immune response, and oxidative stress have been suggested as alternative mechanistic explanations [36]. Hypertension, a representative disease of metabolic syndrome, increases the risk of renal cancer by exerting high mechanical pressure on the renal parenchyma [37,38]. Acid reflux induced by increased abdominal pressure could also increase the risk of esophageal adenocarcinoma [39]. Excessive intake of iodide has been suggested as a cause of thyroid cancer [16]. However, these traditional approaches for the mechanistic explanation of obesity-related cancer have not investigated the significance of intracellular lipid accumulation and metabolic changes in cancer cells. A recent study on nonalcoholic steatohepatitis (NASH) revealed that changes in lipid metabolism caused by ectopic lipid accumulation resulted in hepatocellular carcinoma (HCC) [40]. The mechanism explaining the development of HCC from NASH involves lipid-laden hepatocytes that produce lipid paradoxically; moreover, genes related to lipogenesis, such as SREBPIc, play an important role in this process [41]. As a result, lipid-regulating mechanisms, such as de novo lipogenesis, are important in NASH and cancer development.

Thyroid cancer has also been suggested as an obesity-related cancer according to recent epidemiologic, experimental, and clinical studies $[15,17,19,23-25]$. The mechanism by which obesity can increase the risk of thyroid cancer has remained unelucidated, although excessive intake of iodide has been suggested as one of the causes. Similar to the other internal organs such as the liver and heart, the thyroid gland can also be subject to ectopic fat accumulation, which causes pathological changes in lipid metabolism of the thyroid gland [14,20]. In line with these previous studies, lipid metabolism in normal thyroid follicular cells and thyroid cancer cells might be important research targets to understand thyroid cancer metabolism. In our study, we first investigated the expression levels of $L X R \alpha$ and $L X R \beta$ in human cancers. LXRs are members of the nuclear receptor family of transcription factors and important regulators of lipid and glucose metabolism; they are closely related to peroxisome proliferator activated receptors, farnesoid $\mathrm{X}$ receptors, and retinoid $\mathrm{X}$ receptors, all of which are important players in intracellular lipid metabolism $[42,43]$. Therefore, we postulated that gaining insights into LXR regulation might be an initial step to understand the intracellular lipid metabolism in human cancers. In liver, lung, and colorectal cancers, $L X R \alpha$ mRNA expression was remarkably increased compared to that in other cancers; $L X R \alpha$ expression is restricted to the liver, intestine, lung, and so forth, and is the highest in the liver, hence the name $L X R \alpha$. $L X R \beta$ has been known to be expressed in almost every tissue, and was hence named a ubiquitous receptor $[3,44]$. Interestingly, $L X R \beta$ expression was higher in thyroid cancer than in other types of cancers, suggesting that intracellular lipid metabolism in thyroid cancer might play an important role in tumor behavior. Supporting our presumption, the clinicopathological analyses according to $L X R \beta$ expression status revealed that high $L X R \beta$ expression was correlated with high frequency of male gender and advanced TNM stages although their $P$ values were marginal. In addition, the molecular features of the group with high $L X R \beta$ expression revealed interesting findings. Although the prevalence of $B R A F$ V600E mutation was not different, high $L X R \beta$ expression was correlated with a high mRNA cluster number, a lower BRAF/RAS score and a lower differentiation score, which implied a more aggressive and de-differentiated state. Thus, the characteristics of lipid metabolism might have a prognostic and biological impact on thyroid cancer, independent of the type of driver mutation.

Although we could not verify the mechanistic link between $L X R \beta$ and ribosomes, GSEA and our validation experiments clearly showed the coordinate correlation between $L X R \beta$ and ribosome-related gene sets. Traditionally, lipid metabolism in cancer has been known to be involved in the synthesis of cell membranes essential for cell proliferation [45]. Our data indicating the close relationship of $L X R \beta$ with ribosome, suggested that increased lipid metabolism might be coupled with increased rate of protein synthesis, which is also essential process of cancer cell proliferation. Taken together, increased $L X R \beta$ expression might indicate that cancer cells were in high proliferative activity.

In fact, we analyzed overall survival and recurrence rate according to $L X R \beta$ expression status but unfortunately could not find any significant difference between high and low $L X R \beta$ expression groups (data not shown). This data indicated that high $L X R \beta$ expression could not be used as a useful prognostic factor. However, as we described above, because clinical and biological features of high $L X R \beta$ expression group consistently showed aggressive features, the prognostic impact of $L X R \beta$ expression needs further study in the future. In this study, we used American Joint Committee on Cancer (AJCC) 7th edition to analyze clinicopathological features of TCGA THCA although individual pathologic reports could be obtained as source files. This might be the limitation of this paper to understand the prognostic impact of $L X R \beta$ expression according to the currently used AJCC 8th edition.

These data suggest that lipid metabolism in thyroid cancer might be closely related to translational activity which is a 
known signature of aggressive tumors. In future, the crosslink between lipid metabolism and master regulators of ribosomal activity, such as mammalian target of rapamycin (mTOR)/S6K pathways, might be an interesting research topic in thyroid cancer metabolism [46-48].

\section{CONFLICTS OF INTEREST}

No potential conflict of interest relevant to this article was reported.

\section{ACKNOWLEDGMENTS}

This study was supported by the National Research Foundation of Korea (NRF) grant funded by the Korea government (MSIT) (NRF-2020R1A2C1006047 to Jandee Lee) and (NRF-2018R1A2B6004179 to Young Suk Jo).

\section{AUTHOR CONTRIBUTIONS}

Conception or design: J.L., Y.S.J. Acquisition, analysis, or interpretation of data: S.J., H.K., M.J.C. Drafting the work or revising: S.J., I.K.K., J.L., Y.S.J. Final approval of the manuscript: S.J., I.K.K., H.K., M.J.C., J.L., Y.S.J.

\section{ORCID}

Seonhyang Jeong https://orcid.org/0000-0002-5549-9182

Jandee Lee https://orcid.org/0000-0003-4090-0049

Young Suk Jo https://orcid.org/0000-0001-9926-8389

\section{REFERENCES}

1. Woolner LB, Beahrs OH, Black BM, Mcconahey WM, Keating FR Jr. Classification and prognosis of thyroid carcinoma. A study of 885 cases observed in a thirty year period. Am J Surg 1961;102:354-87.

2. Kim TH, Kim YN, Kim HI, Park SY, Choe JH, Kim JH, et al. Prognostic value of the eighth edition AJCC TNM classification for differentiated thyroid carcinoma. Oral Oncol 2017;71: 81-6.

3. Jung KW, Won YJ, Kong HJ, Oh CM, Seo HG, Lee JS. Cancer statistics in Korea: incidence, mortality, survival and prevalence in 2010. Cancer Res Treat 2013;45:1-14.

4. Hughes DT, Haymart MR, Miller BS, Gauger PG, Doherty GM. The most commonly occurring papillary thyroid can- cer in the United States is now a microcarcinoma in a patient older than 45 years. Thyroid 2011;21:231-6.

5. Ito Y, Miyauchi A, Oda H. Low-risk papillary microcarcinoma of the thyroid: a review of active surveillance trials. Eur J Surg Oncol 2018;44:307-15.

6. Lim H, Devesa SS, Sosa JA, Check D, Kitahara CM. Trends in thyroid cancer incidence and mortality in the United States, 1974-2013. JAMA 2017;317:1338-48.

7. Qian ZJ, Jin MC, Meister KD, Megwalu UC. Pediatric thyroid cancer incidence and mortality trends in the United States, 1973-2013. JAMA Otolaryngol Head Neck Surg 2019;145:617-23.

8. Albi E, Cataldi S, Lazzarini A, Codini M, Beccari T, Ambesi-Impiombato FS, et al. Radiation and thyroid cancer. Int J Mol Sci 2017;18:911.

9. Zidane M, Cazier JB, Chevillard S, Ory C, Schlumberger M, Dupuy $\mathrm{C}$, et al. Genetic susceptibility to radiation-related differentiated thyroid cancers: a systematic review of literature. Endocr Relat Cancer 2019;26:R583-96.

10. Ito Y, Yoshida H, Maruo R, Morita S, Takano T, Hirokawa $\mathrm{M}$, et al. BRAF mutation in papillary thyroid carcinoma in a Japanese population: its lack of correlation with high-risk clinicopathological features and disease-free survival of patients. Endocr J 2009;56:89-97.

11. Sandler JE, Huang H, Zhao N, Wu W, Liu F, Ma S, et al. Germline variants in DNA repair genes, diagnostic radiation, and risk of thyroid cancer. Cancer Epidemiol Biomarkers Prev 2018;27:285-94.

12. Yamamoto H, Hayashi K, Scherb H. Association between the detection rate of thyroid cancer and the external radiation dose-rate after the nuclear power plant accidents in Fukushima, Japan. Medicine (Baltimore) 2019;98:e17165.

13. Jo YS, Li S, Song JH, Kwon KH, Lee JC, Rha SY, et al. Influence of the BRAF V600E mutation on expression of vascular endothelial growth factor in papillary thyroid cancer. J Clin Endocrinol Metab 2006;91:3667-70.

14. Sari R, Balci MK, Altunbas H, Karayalcin U. The effect of body weight and weight loss on thyroid volume and function in obese women. Clin Endocrinol (Oxf) 2003;59:258-62.

15. Renehan AG, Tyson M, Egger M, Heller RF, Zwahlen M. Body-mass index and incidence of cancer: a systematic review and meta-analysis of prospective observational studies. Lancet 2008;371:569-78.

16. Xing M, Alzahrani AS, Carson KA, Viola D, Elisei R, Bendlova B, et al. Association between BRAF V600E mutation and mortality in patients with papillary thyroid cancer. JAMA 
2013;309:1493-501.

17. Son H, Lee H, Kang K, Lee I. The risk of thyroid cancer and obesity: a nationwide population-based study using the Korea National Health Insurance Corporation cohort database. Surg Oncol 2018;27:166-71.

18. Kwon H, Chang Y, Cho A, Ahn J, Park SE, Park CY, et al. Metabolic obesity phenotypes and thyroid cancer risk: a cohort study. Thyroid 2019;29:349-58.

19. Kitahara CM, Platz EA, Freeman LE, Hsing AW, Linet MS, Park Y, et al. Obesity and thyroid cancer risk among U.S. men and women: a pooled analysis of five prospective studies. Cancer Epidemiol Biomarkers Prev 2011;20:464-72.

20. Lee MH, Lee JU, Joung KH, Kim YK, Ryu MJ, Lee SE, et al. Thyroid dysfunction associated with follicular cell steatosis in obese male mice and humans. Endocrinology 2015; 156:1181-93.

21. Lee J, Yi S, Kang YE, Kim HW, Joung KH, Sul HJ, et al. Morphological and functional changes in the thyroid follicles of the aged murine and humans. J Pathol Transl Med 2016; 50:426-35.

22. Kim WG, Park JW, Willingham MC, Cheng SY. Diet-induced obesity increases tumor growth and promotes anaplastic change in thyroid cancer in a mouse model. Endocrinology 2013;154:2936-47.

23. Kim WG, Cheng SY. Mechanisms linking obesity and thyroid cancer development and progression in mouse models. Horm Cancer 2018;9:108-16.

24. Lee J, Lee CR, Ku CR, Kang SW, Jeong JJ, Shin DY, et al. Association between obesity and BRAFV600E mutation status in patients with papillary thyroid cancer. Ann Surg Oncol 2015;22 Suppl 3:S683-90.

25. Gasior-Perczak D, Palyga I, Szymonek M, Kowalik A, Walczyk A, Kopczynski J, et al. The impact of BMI on clinical progress, response to treatment, and disease course in patients with differentiated thyroid cancer. PLoS One 2018;13: e0204668.

26. Hong JH, Yi HS, Yi S, Kim HW, Lee J, Kim KS. Implications of oncocytic change in papillary thyroid cancer. Clin Endocrinol (Oxf) 2016;85:797-804.

27. Apfel R, Benbrook D, Lernhardt E, Ortiz MA, Salbert G, Pfahl M. A novel orphan receptor specific for a subset of thyroid hormone-responsive elements and its interaction with the retinoid/thyroid hormone receptor subfamily. Mol Cell Biol 1994;14:7025-35.

28. Janowski BA, Willy PJ, Devi TR, Falck JR, Mangelsdorf DJ. An oxysterol signalling pathway mediated by the nucle- ar receptor LXR alpha. Nature 1996;383:728-31.

29. Edwards PA, Kennedy MA, Mak PA. LXRs: oxysterol-activated nuclear receptors that regulate genes controlling lipid homeostasis. Vascul Pharmacol 2002;38:249-56.

30. Forman BM, Ruan B, Chen J, Schroepfer GJ Jr, Evans RM. The orphan nuclear receptor LXRalpha is positively and negatively regulated by distinct products of mevalonate metabolism. Proc Natl Acad Sci U S A 1997;94:10588-93.

31. Schultz JR, Tu H, Luk A, Repa JJ, Medina JC, Li L, et al. Role of LXRs in control of lipogenesis. Genes Dev 2000;14: 2831-8.

32. Girish V, Vijayalakshmi A. Affordable image analysis using NIH Image/ImageJ. Indian J Cancer 2004;41:47.

33. Calkin AC, Tontonoz P. Transcriptional integration of metabolism by the nuclear sterol-activated receptors LXR and FXR. Nat Rev Mol Cell Biol 2012;13:213-24.

34. Mokdad AH, Bowman BA, Ford ES, Vinicor F, Marks JS, Koplan JP. The continuing epidemics of obesity and diabetes in the United States. JAMA 2001;286:1195-200.

35. Renehan AG, Frystyk J, Flyvbjerg A. Obesity and cancer risk: the role of the insulin-IGF axis. Trends Endocrinol Metab 2006; 17:328-36.

36. Stone TW, McPherson M, Gail Darlington L. Obesity and cancer: existing and new hypotheses for a causal connection. EBioMedicine 2018;30:14-28.

37. Chow WH, Gridley G, Fraumeni JF Jr, Jarvholm B. Obesity, hypertension, and the risk of kidney cancer in men. N Engl J Med 2000;343:1305-11.

38. Sanfilippo KM, McTigue KM, Fidler CJ, Neaton JD, Chang Y, Fried LF, et al. Hypertension and obesity and the risk of kidney cancer in 2 large cohorts of US men and women. Hypertension 2014;63:934-41.

39. Smith KJ, O'Brien SM, Smithers BM, Gotley DC, Webb $\mathrm{PM}$, Green AC, et al. Interactions among smoking, obesity, and symptoms of acid reflux in Barrett's esophagus. Cancer Epidemiol Biomarkers Prev 2005;14(11 Pt 1):2481-6.

40. Michelotti GA, Machado MV, Diehl AM. NAFLD, NASH and liver cancer. Nat Rev Gastroenterol Hepatol 2013; 10:656-65.

41. Postic C, Girard J. Contribution of de novo fatty acid synthesis to hepatic steatosis and insulin resistance: lessons from genetically engineered mice. J Clin Invest 2008;118:829-38.

42. Seol W, Choi HS, Moore DD. Isolation of proteins that interact specifically with the retinoid $\mathrm{X}$ receptor: two novel orphan receptors. Mol Endocrinol 1995;9:72-85.

43. Yoshikawa T, Shimano H, Amemiya-Kudo M, Yahagi N, 
Hasty AH, Matsuzaka T, et al. Identification of liver X receptor-retinoid $\mathrm{X}$ receptor as an activator of the sterol regulatory element-binding protein 1c gene promoter. Mol Cell Biol 2001;21:2991-3000.

44. Song C, Kokontis JM, Hiipakka RA, Liao S. Ubiquitous receptor: a receptor that modulates gene activation by retinoic acid and thyroid hormone receptors. Proc Natl Acad Sci U S A 1994;91:10809-13.

45. DeBerardinis RJ, Lum JJ, Hatzivassiliou G, Thompson CB. The biology of cancer: metabolic reprogramming fuels cell growth and proliferation. Cell Metab 2008;7:11-20.
46. Mayer C, Grummt I. Ribosome biogenesis and cell growth: mTOR coordinates transcription by all three classes of nuclear RNA polymerases. Oncogene 2006;25:6384-91.

47. Iadevaia V, Huo Y, Zhang Z, Foster LJ, Proud CG. Roles of the mammalian target of rapamycin, mTOR, in controlling ribosome biogenesis and protein synthesis. Biochem Soc Trans 2012;40:168-72.

48. Delarue M, Brittingham GP, Pfeffer S, Surovtsev IV, Pinglay $\mathrm{S}$, Kennedy KJ, et al. mTORC1 controls phase separation and the biophysical properties of the cytoplasm by tuning crowding. Cell 2018;174:338-49. 